\title{
Single Carrier Frequency Division Multiple Access (SC-FDMA) for Filter Bank Multicarrier Communication Systems
}

\author{
Chung Him (George) Yuen, Pooyan Amini, and Behrouz Farhang-Boroujeny \\ ECE department, University of Utah, USA
}

\begin{abstract}
The Third Generation Partnership Project (3GPP) Long Term Evolution (LTE) radio standard has adopted a special form of orthogonal frequency division multiplexing (OFDM) method for the uplink of multiple access networks. This method which is called single carrier frequency division multiple access (SC-FDMA) applies a precoding to each user data set in each OFDM symbol to control its peak-to-average power ratio (PAPR). In this paper, we present a novel formulation of the SC-FDMA and explore possible mimicking the same method when filter bank multicarrier (FBMC) is used for transmission. We find that such direct application of SC-FDMA to FBMC systems is not successful. However, we show that FBMC techniques offer other opportunities that when used correctly lead to a significant reduction in PAPR.
\end{abstract}

\section{INTRODUCTION}

Reducing the peak-to-average power ratio (PAPR) in any transmission system is always desirable as it allows use of more power efficient and cheaper amplifiers at the transmitter. Recently, the Third Generation Partnership Project (3GPP) Long Term Evolution (LTE) radio standard has adopted a special form of orthogonal frequency division multiplexing (OFDM) signaling for the uplink of multiple access networks, called single carrier frequency division multiple access (SCFDMA). SC-FDMA applies a precoding to each user data set in each OFDM symbol to control PAPR, [1], [2], [3]. The standard has recognized this as a critical component to the success of 3GPP LTE products, noting that reducing PAPR directly translates to a lower power consumption and thus a longer battery life of mobile stations.

On the other hand, we note that the adoption of OFDM as a multiple accessing tool has also a number of other short-comings with regard synchronization. In the uplink of a network, the signals from different users should arrive synchronously at the base station. This requires some stringent control and introduces some limitation of the performance of the network. Moreover, additional signal processing steps have to be taken to reduce interference among users as they can never be fully synchronized [4]. The problem of synchronization becomes more serious in cognitive radios where there may be no way of assuring synchronization among primary and secondary users. Noting this, filter bank multicarrier (FBMC) has been proposed as more preferred multicarrier candidate in cognitive radios [5]. This paper, as a step forward in bringing FBMC in par with OFDM, explores possible adaptation of the SC-FDMA to networks that use FBMC signaling for multiple access.

This paper is organized as follows. A novel formulation of SC-FDMA that facilitates a straightforward extension of it to FBMC systems is presented in Section II. The extension of SC-FDMA to FBMC is presented in Section III where it is found that SC-FDMA is incapable of reducing PAPR in this application significantly. Noting this, in Section IV, we present an alternative point of view of SC-FDMA technique that leads to a new idea for single carrier modulation in FBMC systems. This is presented in Section V. Simulation results show excellent PAPR results of this method; almost $4 \mathrm{~dB}$ improvement over SC-FDMA is observed. The concluding remarks are presented in Section VI.

\section{SC-FDMA}

The basic principle of SC-FDMA, in the form adopted in 3GPP LTE standard (i.e., when a set of contiguous subcarriers is allocated to each user), are laid out in [6]. Fig. 1 presents a block diagram of such a SC-FDMA transmitter. A user data stream $s[n]$ is passed through a multiplexer (a serial-to-parallel converter $(\mathrm{S} / \mathrm{P}))$ that divides it into $M$ parallel substreams. In a conventional OFDMA, these substreams are directly allocated to $M$ subcarriers in an OFDM modulator with a total of $N>M$ subcarriers. In SC-FDMA, a precoder is inserted between the multiplexer and OFDM modulator. The precoder takes the $M$ output samples of multiplexer and pre-multiply them with a matrix $P$ of size $L \times M$, where $L \geq M$. The choice of $L>M$ allows addition of some redundancy that are used to further reduce PAPR. It has been noted in [6] that when $L=M$, a good choice of $P$ is the DFT matrix, and when $L>M, P$ is obtained by extending the $M \times M$ DFT matrix vertically and pre-multiplying the resulting $L \times M$ matrix with a diagonal matrix whose diagonal elements form a proper window function. The raised-cosine and square-root raised-cosine window functions have been suggested in [6].

To develop a more in depth understanding of the SC-FDMA, we note that the SC-FDMA transmitter is a multirate digital signal processing system with the data sequence $s[n]$ at its input and the synthesized signal $x[n]$ at its output. Fig. 2 casts this multirate system in a form that will allow its manipulation using the standard multirate signal processing tools. Here, the multiplexer is implemented using a tapped delay line of length $M$ and a set of $M$-fold decimators. $\mathbf{P}$ is the precoder matrix, of size $L \times M$. $\mathbf{E}$ takes the precoder output of length $L$ and 


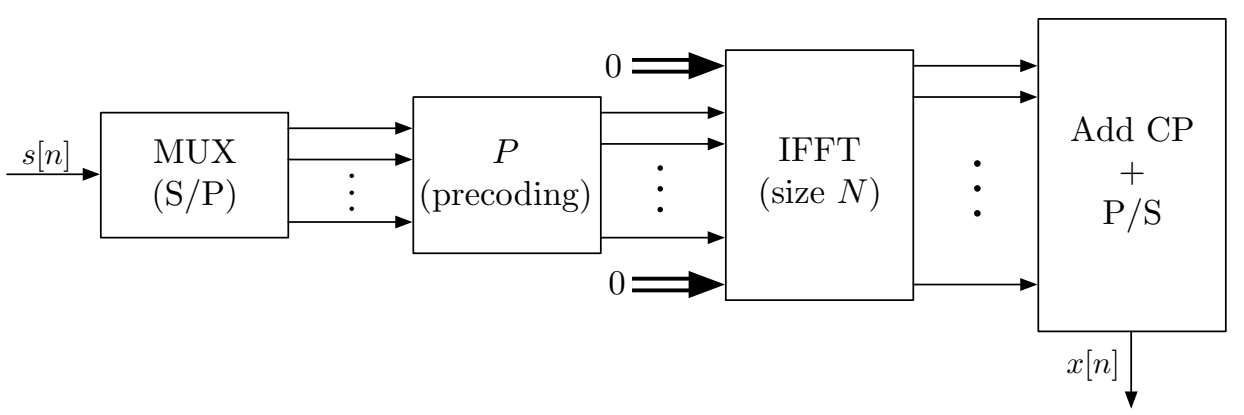

Fig. 1. An SC-FDMA transmitter.

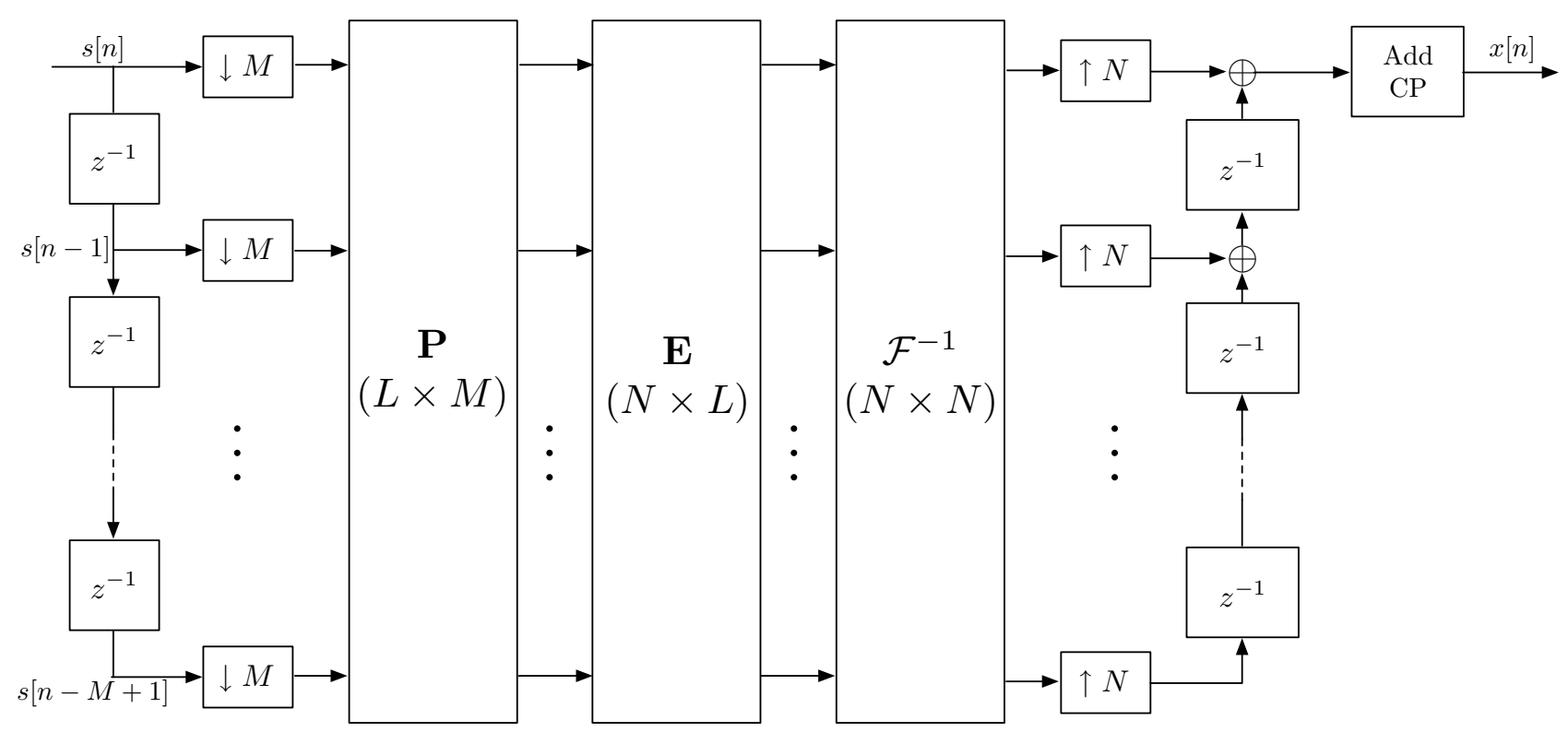

Fig. 2. The SC-FDMA transmitter of Fig. 1 implemented using the standard multirate signal processing blocks.

expand it to the length $N$, by appending zeros to its beginning and its end. The precoded and expanded samples are passed to an IFFT block denoted by $\mathcal{F}^{-1}$. The serial-to-parallel (S/P) block of the structure is implemented using a set of $N$-fold expanders, followed by a set of shift and add operations. The cyclic prefix (CP) samples are added at the end.

Using the standard tools from multirate signal processing, [8], [10], the structure presented in Fig. 2 may be converted to Fig. 3. The transfer function between the single input $s[n]$ and multiple outputs $x_{0}[n]$ through $x_{N-1}[n]$ is thus obtain as

$$
\mathbf{H}(z)=\frac{\mathbf{X}(z)}{S(z)}=\mathbf{G z}
$$

where $\mathbf{G}=\mathcal{F}^{-1} \mathbf{E P}$ and $\mathbf{z}$ is a tapped delay line vector of compatible length. Here,

$$
\mathbf{z}=\left[\begin{array}{llll}
1 & z^{-1} & \cdots & z^{-(M-1)}
\end{array}\right]^{\mathrm{T}}
$$

where the superscript $\mathrm{T}$ denotes matrix or vector transpose. Alternatively, one may note that the vector of impulse response between the input $s[n]$ and the $k$ th output $x_{k}[n]$ is the $k$ th row of $\mathbf{G}$.
Each decimator selects one out of every $M$ samples of each of the sequences $x_{0}[n]$ through $x_{N-1}[n]$, and the expanders followed by the shift and add blocks effectively construct a S/P that sequentially sends the selected samples to the transmitter output. Accordingly, the PAPR of the SC-FDMA transmitter may be calculated as

$$
\text { PAPR }=\frac{\max _{k, n}\left|x_{k}[n]\right|^{2}}{\operatorname{avg}\left[\left|x_{k}[n]\right|^{2}\right]}
$$

where $\operatorname{avg}\left[\left|x_{k}[n]\right|^{2}\right]$ is the average of $\left|x_{k}[n]\right|^{2}$ over all values of $k$ and $n$.

From the above discussion, one finds that the PAPR of SCFDMA will be small, if each row of $\mathbf{G}$ has only one significant element, and the significant elements from different rows have amplitudes that are about the same. The ideal case is when each row of $\mathbf{G}$ has one non-zero element and the non-zero elements from all rows have the same magnitude.

From the developments in [6] one may infer that $\mathbf{G}$ fulfills the above desirable properties, to some extent, if $\mathbf{P}$ is a DFT matrix of size $M \times M$. Moreover, a better conditioned $\mathbf{G}$ 


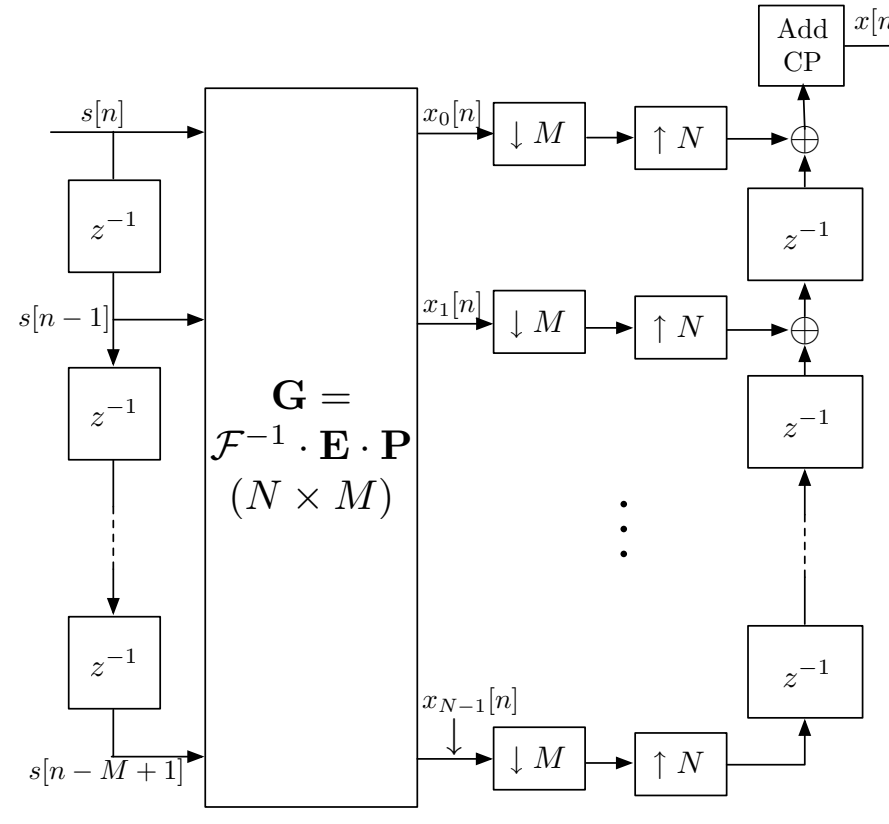

Fig. 3. A simplified version of the SC-FDMA of Fig. 2.

is obtained if $\mathbf{P}$ is constructed by cyclically extending the DFT matrix vertically (i.e., extend $\mathbf{P}$ from size $M \times M$ to $L \times M$, with $L>M$ ) and applying a roll-off window to each of its columns. Clearly, here, there is a penalty that one has to pay for reducing PAPR - the number of subcarriers used for transmission of $M$ QAM symbols of a user will increase from $M$ to $L$.

\section{SC-FDMA EXTENSION TO FBMC}

The SC-FDMA structure presented in Fig. 2 may be thought as a special case of a more general structure when the precoder is an analysis filter bank with a prototype filter $H^{\mathrm{a}}(z)$ and the multicarrrier modulator is a synthesis filter bank with a prototype filter $H^{\mathrm{s}}(z)$. Fig. 4 presents such a generalization, where $E_{k}^{\mathrm{a}}(z)$ and $E_{k}^{\mathrm{s}}(z)$ are the polyphase components associated with $H^{\mathrm{a}}(z)$ and $H^{\mathrm{s}}(z)$. The difference between this structure and that of Fig. 2 is the addition of the polyphase components.

Recall from our previous discussions that the choice of the precoder $\mathbf{P}$ results in a well conditioned matrix $\mathbf{G}$ with only one significant element in each of its rows and this leads to a low PAPR. Unfortunately, the presence of the polyphase components $E_{k}^{\mathrm{a}}(z)$ and $E_{k}^{\mathrm{s}}(z)$ results in a set of impulse responses between the input and the points prior to the $N$-fold expanders that will not be as well conditioned as those associated with $\mathbf{G}$. This results in some deterioration of PAPR. To quantify this, Fig. 5 compares the cumulative complementary distribution functions (CCDF) of a user signal where 128 contiguous subcarriers out of 512 subcarriers are used. CCDF is a probability function and is defined as the probability of PAPR being greater than the value specified on the horizontal axis. CCDF curves are presented for the cases when (i) there is no precoder, (ii) SC-FDMA is applied to an OFDM system and the precoder is a DFT matrix (DFTpOFDM), (iii) and (iv) SC-FDMA is applied to an FBMC system and precoder is either a DFT or a filter bank. These are labeled DFTp-SMT and FBp-SMT, where SMT refers to the staggered multitone modulation. SMT, which in many publications is referred as OFDM/OQAM, is the most widely studied FBMC technique in the literature, [11]. Also presented in Fig. 5 is the result of the single carrier FBMC that is developed in the subsequent sections of this paper. The FBMC uses a square-root prototype filter with roll-off factor $\alpha=1$. The design method proposed in [9] has been used to design the prototype filter.

The above results and similar numerical results presented in [7], clearly, show that mimicking the SC-FDMA developed for OFDMA to FBMC is not as effective in reducing PAPR.

\section{FILTERING INTERPRETATION OF SC-FDMA}

An alternative way of looking at SC-FDMA of Fig. 1 that includes both OFDM and FBMC is presented in Fig. 6. This structure contains $M$ parallel branches, each consisting of an analysis filter, $H_{k}^{\mathrm{a}}(z)$, an $M$-fold decimator, an $N$ fold expander, and a synthesis filter, $H_{k}^{\mathrm{a}}(z)$. Following a few standard steps from the theory of multirate signal processing [8], one finds the z-transform of $x[n]$ as

$$
X(z)=\sum_{k=0}^{M-1} A_{k}(z) S\left(z^{K} W_{M}^{k}\right)
$$

where $K=N / M$ and $W_{M}=e^{-j 2 \pi / M}$ and

$$
A_{k}(z)=\frac{1}{M} \sum_{k=0}^{M-1} H_{k}^{\mathrm{a}}\left(z^{K} W_{M}^{k}\right) H_{k}^{\mathrm{s}}(z) .
$$

If one could design the pair of analysis and synthesis filters such that $A_{k}(z)=0$, for $k \neq 0$, (4) would simplify to

$$
X(z)=A_{0}(z) S\left(z^{L}\right) .
$$

This result has the following interpretation. The output sequence $x[n]$ is an interpolated version of the input data sequence $s[n]$ with the interpolation factor $K=N / M$ and interpolation filter $A_{0}(z)$. Hence, when $s[n]$ is from a specific/known constellation, the PAPR will be a function of $A_{0}(z)$.

The approach taken in [6] to reduce PAPR by extending the DFT matrix and applying a roll-off window (discussed earlier in this paper), in fact, serves the purpose of introducing a rolloff to $A_{0}(z)$ and also forcing $A_{k}(z) \approx 0$, for $k \neq 0$. However, unfortunately, the results presented in Fig. 5 imply that such methods are less effective in FBMC systems.

\section{Single CARrier For FBMC Systems}

Following the above observations, in this section, we concentrate on adopting a novel method for signal carrier modulation in FBMC systems. We note that the presence of the $M$-fold decimator will introduce $M$ replications of the spectrum of $s[n]$ and, as a results, significant aliasing terms 


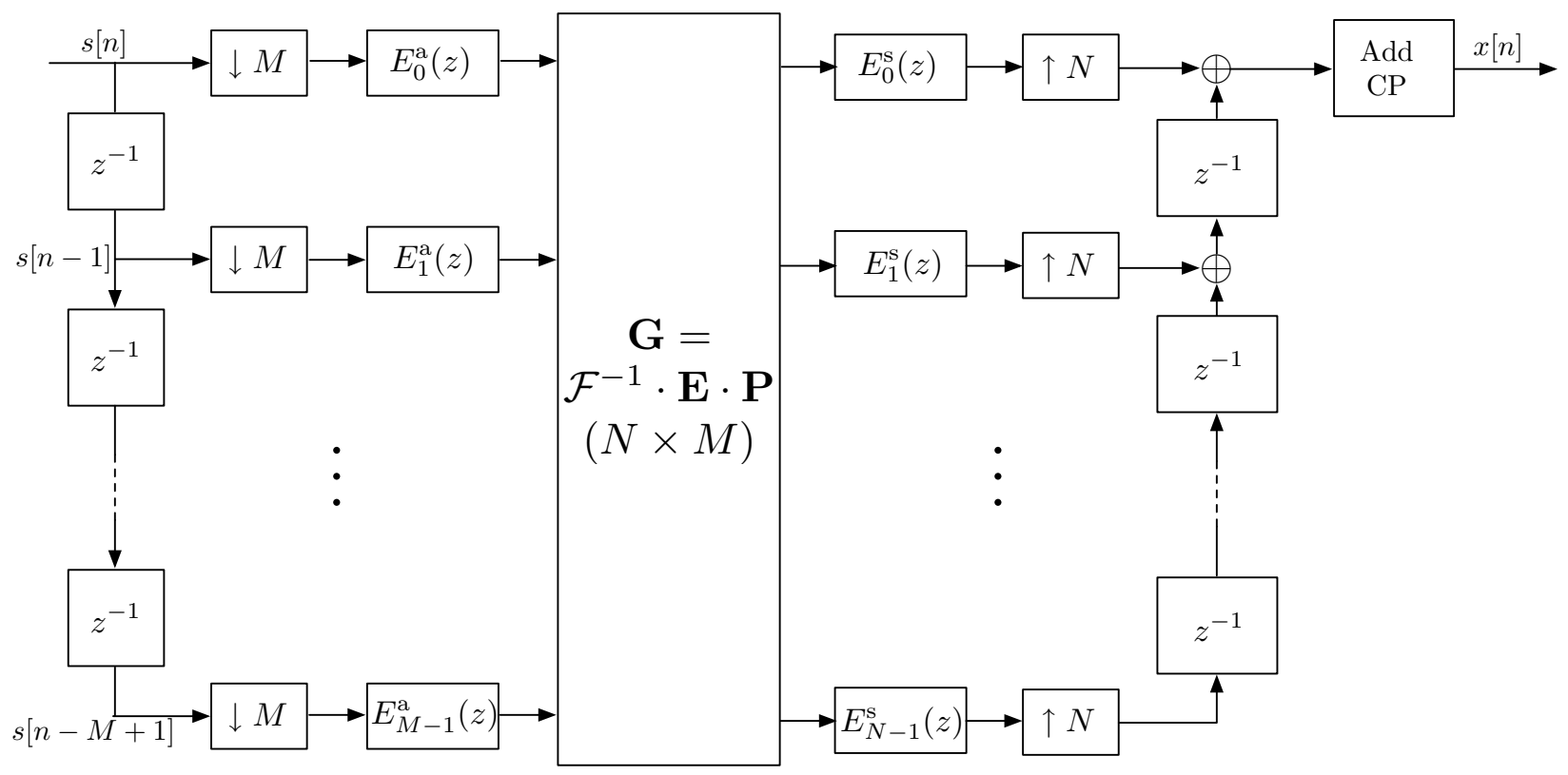

Fig. 4. A polyphase block diagram of SC-FB. This reduces to Fig. 2 when the polyphase components $E_{k}^{\mathrm{a}}(z)$ and $E_{k}^{\mathrm{s}}(z)$ are all equal to 1 .

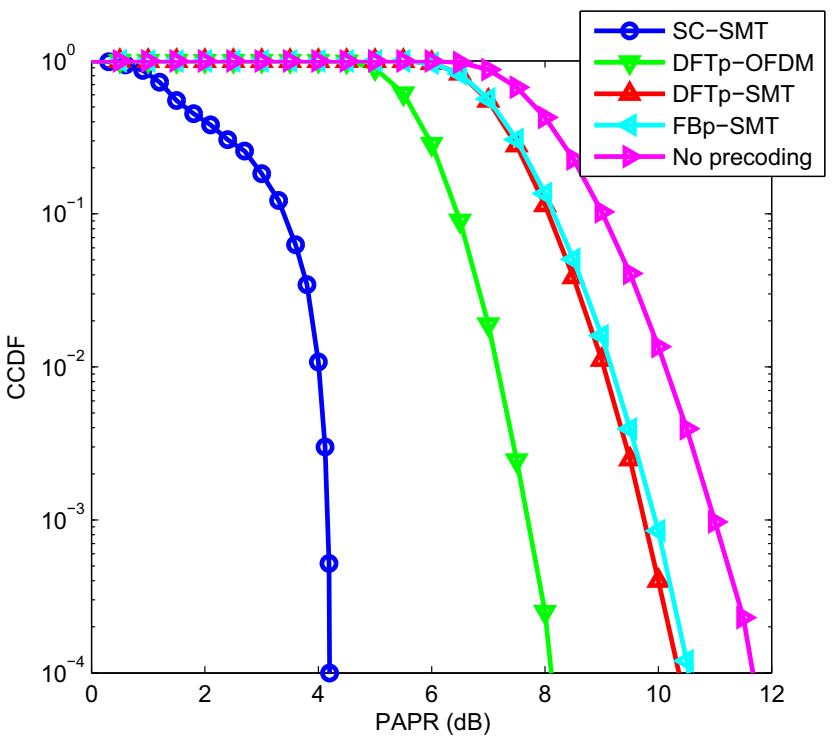

Fig. 5. Cumulative complementary distribution functions of different modulation methods.

may be introduced in the output sequence $x[n]$, as expressed mathematically in (4).

A natural method of removing the aliasing components, is to remove the $M$-fold decimators from the structure of Fig. 6 and instead replace each pair of decimator-expander by an $K$-fold expander, where, as defined before, $K=N / M$.

Recalling

$$
A_{0}(z)=\frac{1}{M} \sum_{k=0}^{M-1} H_{k}^{\mathrm{a}}\left(z^{K}\right) H_{k}^{\mathrm{s}}(z),
$$

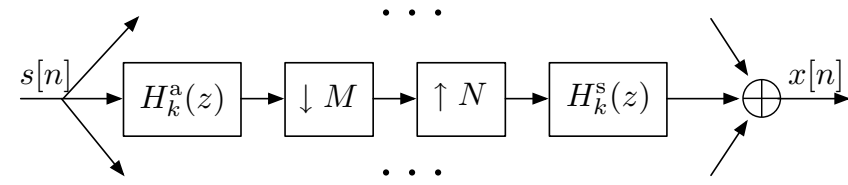

Fig. 6. The SC-FDMA transmitter presented as a parallel set branches filtering decimator and expanders.

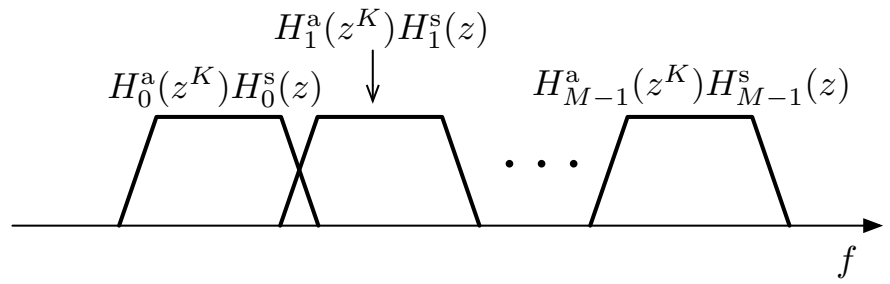

Fig. 7. The SC-FDMA transmitter presented as a parallel set branches filtering decimator and expanders.

and assuming that $H_{k}^{\mathrm{a}}(z)$ and $H_{k}^{\mathrm{s}}(z)$ are square-root Nyquist filters, one will find that the terms under summation in (7) are Nyquist filters and when the subcarriers are contiguous (as in the case of SC-FDMA in 3GPP LTE) they add up to a flat passband. Fig. 7 depicts this concept. $A_{0}(z)$ is thus a is a passband filter with transition bands that resemble that of a Nyquist filter with a roll-off factor $\alpha / M$, where $\alpha$ is the roll-off factor of the prototype filters used for $H_{k}^{\mathrm{a}}(z)$ and $H_{k}^{\mathrm{s}}(z)$. Noting that in most practical implementations of SMT (OFDM/OQAM), $\alpha=1$ is used, we conclude that $A_{0}(z)$ is a Nyquist filter with a normalized bandwidth of $M / N=1 / K$, roll-off factor $1 / M$, and modulated to desired position within the multiple access band. Moreover, 
considering the fact that modulation in SMT is offset QAM (OQAM), we propose the structure presented in Fig. 8. In this

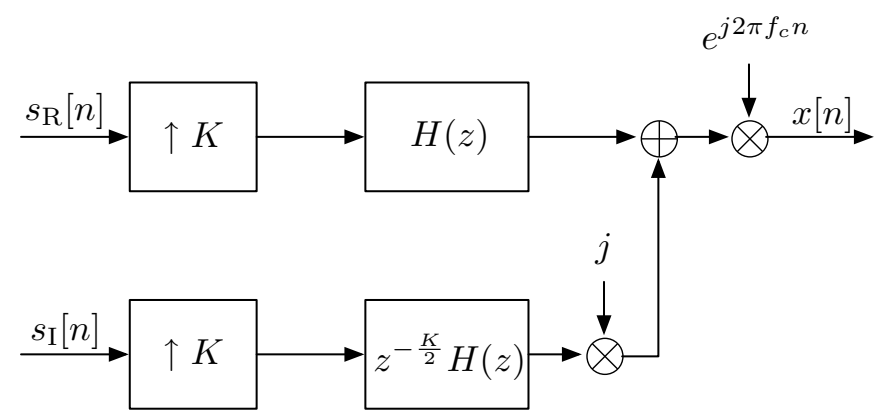

Fig. 8. The SC-FB structure of a transmitter in the uplink of and FBMCbased multiple access network.

structure $H(z)$ is a Nyquist filter in which the zero crossings of its impulse response occur at an interval of $K$ samples. The inputs $s_{\mathrm{R}}[n]$ and $s_{\mathrm{I}}[n]$ are the real and imaginary parts of $s[n]$. The additional factor $z^{-\frac{K}{2}}$ is to introduce an offset of half a symbol interval between the phase and quadrature parts of each symbol. Finally, the multiplication by $e^{j 2 \pi f_{c} n}$ shifts the generated OQAM signal to the desired part of the multiple access band.

The CCDF result of this modulator is presented in Fig. 5 along with those of SC-FDMA proposed in 3GPP LTE standard and other methods that were discussed earlier. As noted before, direct mimicking of the 3GPP LTE proposed SCFDMA in an FBMC-based system leads to an inferior PAPR as compared to OFDM. However, as the results presented in Fig. 5 show, the single carrier modulation that we propose here results in a PAPR which is almost $4 \mathrm{~dB}$ better than SC-FDMA proposed in 3GPP LTE standard for OFDM-based systems. Nevertheless, one should note that the PAPR of SC-FDMA can be improved by adding a window at the output of the precoder, [6]. But, this would be at the cost of some loss in bandwidth efficiency. The results presented in Figure 2 of [2] (which uses the same parameters as here) indicate that to achieve a PAPR comparable to the proposed method here, an roll-off factor $\alpha=0.6$ should be applied to SC-FDMA. This is an additional $60 \%$ in bandwidth usage.

We note that part of the reason that the PAPR of the proposed SC-SMT is significantly lower than the PAPR in SC-FDMA is related to the fact that it is based on OQAM modulation. The off-set introduced between the phase and quadrature components of each symbol distributes the power more homogeneously within each symbol period. We also note that the single carrier nature of the transmitted signal in SC-SMT does not avoid the possibly of using a multicarrier demodulator as in SC-FDMA. Subcarrier analysis and per subcarrier equalization proposed in 3GPP LTE standard are directly applicable to single carrier modulated SMT method.

\section{CONCLUSION}

We presented a study of SC-FDMA system proposed by 3GPP LTE standard committee. We also explored a direct mimicking the SC-FDMA to an FBMC-based network. Our study revealed that such method does not lead to a satisfactory performance. It also revealed the reasons behind why mimicking SC-FDMA to FBMC-based systems has inferior performance when compared to OFDM-based systems. This understanding of SC-FDMA led us to propose a novel single carrier modulation for FBMC systems. The proposed system can still benefit from the frequency domain equalization that is proposed in 3GPP LTE. In addition, similar to previous reports on FBMC, e.g., [5], one can still argue that the superior confinement of the spectra of different users within different bands makes FBMC an excellent choice for cognitive radios.

\section{REFERENCES}

[1] G., Berardinelli, L.A. Ruiz de Temino, S. Frattasi, M. Rahman, and P. Mogensen, "OFDMA vs. SC-FDMA: performance comparison in local area imt-a scenarios," IEEE Wireless Communications, vol. 15, no. 5, pp. 64 - 72, Oct. 2008 .

[2] G. Huang, A. Nix, and S. Armour, "Impact of Radio Resource Allocation and Pulse Shaping on Papr of SC-FDMA Signals," IEEE 18th International Symposium on Personal, Indoor and Mobile Radio Communications, PIMRC 2007. pp. 1 - 5, 2007.

[3] M. Rinne, M. Kuusela, E. Tuomaala, P. Kinnunen, I. Kovacs, K. Pajukoski, and J. Ojala, "A Performance Summary of the Evolved 3G (E-UTRA) for Voice Over Internet and Best Effort Traffic," IEEE Transactions on Vehicular Technology, vol. 58, no. 7, pp. 3661 - 3673, Sept. 2009.

[4] M. Morelli, C.-C. Jay Kuo, and M.-O. Pun, "Synchronization techniques for orthogonal frequency division multiple access (OFDMA): A tutorial review," Proceedings of IEEE, vol. 95, no. 7, July 2007, pp. 1394-1427.

[5] B. Farhang-Boroujeny and R. Kempter, "Multicarrier communication techniques for spectrum sensing and communication in cognitive radios," IEEE Communications Magazine, Special Issue on Cognitive Radios for Dynamic Spectrum Access, vol. 46, no 4, April 2008, pp. 80-85.

[6] S.B. Slimane, "Reducing the Peak-to-Average Power Ratio of OFDM Signals Through Precoding," IEEE Transactions on Vehicular Technology, vol. 56, no. 2, pp. 686 - 695, March 2007.

[7] T. Ihalainen, A. Viholainen, T. H. Stitz, M. Renfors, and M. Bellanger, "Filter Bank Based Multi-Mode Multiple Access Scheme for Wireless Uplink," Proceedings of the 17th European Signal Processing Conference, Aug. 24-28, Glascow, Scotland, pp. 1354-1358.

[8] P.P. Vaidyanathan, Multirate Systems and Filter Banks. Englewood Cliffs, New Jersey, Prentice Hall, 1993.

[9] B. Farhang-Boroujeny, "A square-root Nyquist (M) filter design for digital communication systems," IEEE Trans. on Signal Proc., vol. 56, no. 5, May 2008, pp. 2127-2132.

[10] B. Farhang-Boroujeny, Signal processing techniques for software radios, Lulu publishing (http://www.lulu.com/), 2008.

[11] B. Farhang-Boroujeny and C.H. (George) Yuen, "Cosine Modulated and Offset QAM Filter Bank Multicarrier Techniques: A ContinuousTime Prospect,' Eurasip Journal on Advances in Signal processing, Volume 2010 (2010), Special Issue on Filter Banks for Next-Generation Multicarrier Wireless Communications, Article ID 165654, 16 pages. 